\title{
Application of the prone position in myomectomy by transvaginal natural orifice transluminal endoscopic surgery
}

\author{
Lu Huang, Li He, Ling Zhang, Xiaoqin Gan, Jigang Jia, Yue Yang, Yonghong Lin \\ Department of Gynecology, Chengdu Women's and Children's Central Hospital, School of Medicine, University of Electronic Science \\ and Technology of China, Chengdu, Sichuan, China
}

Videosurgery Miniinv DOI: https://doi.org/10.5114/wiitm.2020.95397

\begin{abstract}
Introduction: Natural orifice transluminal endoscopic surgery (NOTES) is a new concept of minimally invasive surgery. It could prevent complications related to the trocar in laparoscopic surgery, and help achieve ideal cosmetic outcomes.

Aim: To describe the safety and feasibility of the prone position in transvaginal NOTES (V-NOTES) resection of posterior uterine myoma.

Material and methods: Seventeen patients were included in the study from February to December 2019. All the patients were diagnosed with solitary posterior uterine myoma and underwent V-NOTES myomectomy in the prone position. We measured the characteristics and the surgical outcomes of these patients, to evaluate the safety and feasibility of the prone position in V-NOTES myomectomy.

Results: The mean age of the patients was $38.71 \pm 7.68$ years and the mean body mass index was $22 \pm 2.02 \mathrm{~kg} / \mathrm{m}^{2}$. Five patients had a cesarean section once, and 1 patient had a history of two operations (cesarean section and laparoscopic cholecystectomy). The remaining patients had no history of surgery. The mean myoma volume in the ultrasound report was $121.99 \pm 125.24 \mathrm{~cm}^{3}$. The mean operation time was $107.48 \pm 34.16 \mathrm{~min}$. The mean hemoglobin decrease $48 \mathrm{~h}$ after the operation was $1.37 \pm 0.66 \mathrm{~g} / \mathrm{dl}$. The mean weight of the myoma was $183.88 \pm 144.29 \mathrm{~g}$. The mean VAS score $12 \mathrm{~h}$ and $24 \mathrm{~h}$ after surgery was $2 \pm 0.87$ and $1.18 \pm 0.73$, respectively. The mean postoperative hospital stay was $3.18 \pm 0.39$ days. One patient was converted to TU-LESS. No other complications such as massive hemorrhage, infection or injury occurred.

Conclusions: The prone position in V-NOTES myomectomy is safe and feasible. It expands the operative space and reduces the difficulty of surgery. Perhaps it can be used as a standard position for posterior uterine myomectomy by V-NOTES.
\end{abstract}

Key words: myomectomy, prone position, transvaginal natural orifice transluminal endoscopic surgery.

\section{Introduction}

Natural orifice transluminal endoscopic surgery (NOTES) is defined as an operation method that uses the natural orifices of the body surface, such as the mouth, anus, vagina or urethra, as surgical channels of endoscopy to avoid incision scars on the abdominal wall [1]. It could also prevent complications related to the trocar in laparoscopic surgery, and help achieve ideal cosmetic outcomes.

During the past decade, with its use flourishing in general surgery and urologic surgery, NOTES has emerged as a new concept of minimally invasive surgery. Recently, transvaginal natural orifice translu-

\section{Address for correspondence}

Dr. Yonghong Lin. Department of Gynecology, Chengdu Women's and Children's Central Hospital, School of Medicine, University

of Electronic Science and Technology of China, 1617 Riyue Avenue, Chengdu 611731, Sichuan, China, e-mail: cdsfnetzxyy123@sina.com 
minal endoscopic surgery (V-NOTES) has been performed in many kinds of gynecologic procedures, such as adnexal surgery, hysterectomy and myomectomy. We retrieved the previous published literatures and found that because of the relatively restricted vision and the narrow surgical space, the use of V-NOTES in myomectomy, especially the excision of large uterine fibroids or posterior uterine fibroids, is limited. Therefore, we tried to find a way to make posterior wall myomectomy easier to implement by V-NOTES.

This was a retrospective study. Our hospital began to attempt the prone position in myomectomy by V-NOTES from February 2019. In this study, we collected the medical records of all the cases of V-NOTES myomectomy in the prone position from February to December 2019 (17 cases). In these cases, solitary posterior uterine leiomyoma was detected by transvaginal ultrasonography. The characteristics, perioperative outcomes and complications of the patients were collected in order to prove the safety and feasibility of the prone position in V-NOTES posterior myomectomy.

\section{Aim}

To describe the safety and feasibility of the prone position in V-NOTES resection of posterior uterine myoma.

\section{Material and methods}

The authors confirmed that Institutional Review Board (IRB) approval of our hospital was obtained before the study was started.

The inclusion criteria were as follows: 1 . women older than 18 years; 2. laparoscopic surgery is required for solitary posterior uterine myoma; 3 . the vital signs are stable and can be tolerated by laparoscopy; 4. patients can understand the research program and are willing to participate in this study, providing written informed consent. The excluding criteria were as follows: 1 . acute infection stage, preoperative deep venous thrombosis or hypercoagulability, fasting blood sugar $>11.1 \mathrm{mmol} / \mathrm{l}$, blood pressure > 160/100 mm Hg, liver and kidney dysfunction, mental illness and other surgical contraindications; 2. history of rectal surgery, suspected of rectovaginal septum endometriosis, tumors or severe adhesions; 3. virginity; 4. pregnancy.

Seventeen patients were included in the study from February to December 2019. All the patients were diagnosed with solitary posterior uterine myoma and underwent myomectomy in the prone position by V-NOTES. Two of the seventeen patients were given a red blood cell transfusion before the operation due to anemia (hemoglobin $(\mathrm{Hb}) 6.6 \mathrm{~g} / \mathrm{dl}$ and $7.1 \mathrm{~g} / \mathrm{dl}$, respectively). All operations were performed by experienced gynecological endoscopists. We collected information of the patients about age, height, weight, operation history, myoma volume, operation time, estimated blood loss, preoperative and $48 \mathrm{~h}$ postoperative $\mathrm{Hb}$, weight of myoma, postoperative anal exhaust time, visual analogue scale (VAS) scores $12 \mathrm{~h}$ and $24 \mathrm{~h}$ after surgery, postoperative analgesia and perioperative complications. The myoma volume was calculated with the ellipsoid formula according to the size of ultrasound measurement: $\mathrm{L} \times \mathrm{W} \times \mathrm{D} \times 0.523$, where $L=$ length, $W=$ width, and $D=$ depth [2]. The primary complication was conversion to transumbilical laparo-endoscopic single-site surgery (TU-LESS), conventional laparoscopic surgery or laparotomy. The secondary complications were massive hemorrhage, infection and injury. The VAS score was as follows: $0=$ painless, 1 to $3=$ mild pain, 4 to $6=$ moderate pain, 7 to $10=$ severe pain (the higher the score, the more intense the pain).

\section{Surgical techniques}

Under general anesthesia with endotracheal intubation, the patients were placed in the prone position with legs apart and head to one side to keep the endotracheal intubation unobstructed (Photo 1). Before placing the patient in the prone position, a gel pad should be placed on the operating table to prevent pressure ulcer (Photo 2).

\section{The colpotomy}

A vaginal posterior fornix incision was made and the pouch of Douglas was opened. Then a disposable multiple-instrument access port (Beijing Aerospace Kadi Technology Development Institute, HK-TH-60.4TY) was inserted through the incision into the pelvic cavity (Photos $3 \mathrm{~A}, \mathrm{~B}$ ). A pneumoperitoneum was created up to $14 \mathrm{~mm} \mathrm{Hg}$ of $\mathrm{CO}_{2}$ insufflation (Photo $3 \mathrm{C}$ ). Then patients were placed in the Trendelenburg position to make the bowel retracted.

\section{Operation process}

All the instruments and sutures we used were the same as which we used in conventional laparoscopic 

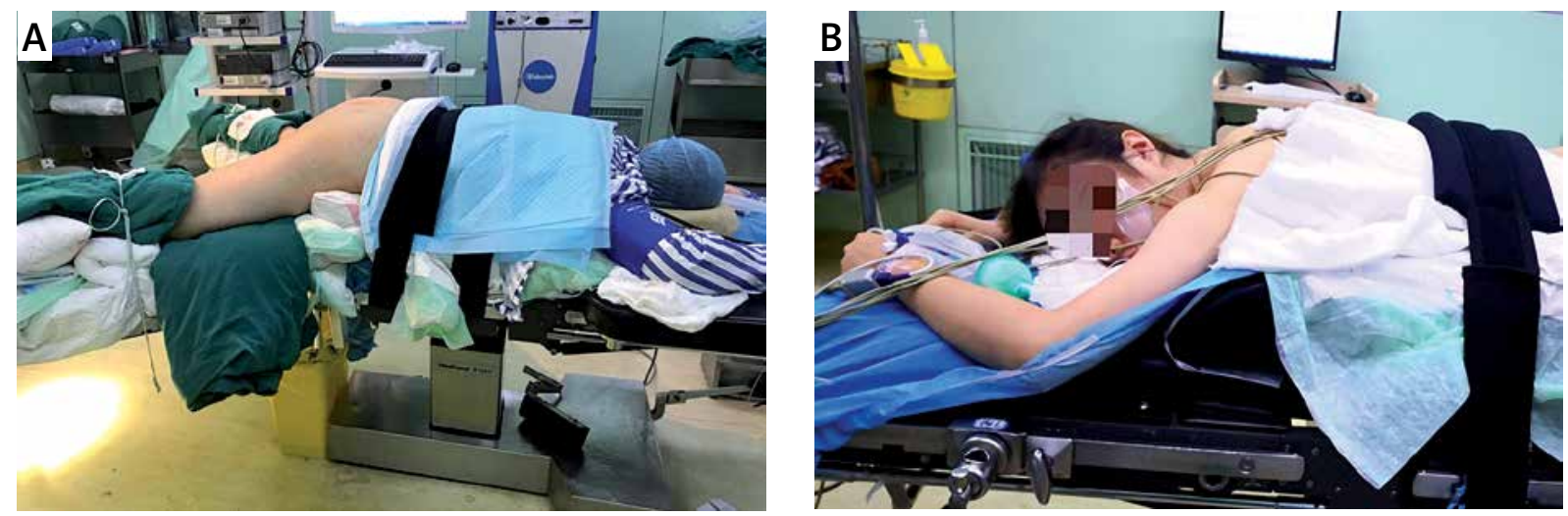

Photo 1. A - Patient in prone position. B - Patient's head to one side to keep endotracheal intubation unobstructed

surgery. We inserted a 10-mm, 30-degree endoscope (Karl Storz GmbH \& Co. KG, Tuttlingen, Germany) to explore the pelvis (Photo 4). In the prone position, laparoscopic access to the pelvic cavity revealed that the location of the posterior wall uterine myoma and the operating space was similar to that of the anterior uterine myoma (Photo 5). After the location of the fibroid was identified, pituitrin $6 \mathrm{u}$ was injected into the myometrium and the protruding site was incised by a unipolar hook. Enucleation of the myoma was performed the same way as conventional laparoscopy (Photo 6). Then the mass was bagged and if necessary the myoma was cut into pieces in the bag. Finally, the bag was removed from the disposable incision retractor intact, to avoid the risk of leiomyosarcoma spread.

The uterine defect was repaired with double layers with 0\# absorbable suture (V-LOC 180 Absorbable Wound Closure Suture by Medtronic USA) (Photo 7).

\section{Completion of the procedures}

Finally, the vaginal wound was closed with 2-0 Vicryl suture. Cefmetazole $1 \mathrm{~g}$ was administered intravenously $30 \mathrm{~min}$ before surgery and again $12 \mathrm{~h}$ apart. All the patients used a patient controlled analgesia (PCA) pump to relieve postoperative pain. Patients had to meet 3 criteria to be discharged: 1) having stayed in an afebrile status for at least $24 \mathrm{~h}$; 2) showing no evidence of surgical complications; 3) results of blood routine test were normal.

\section{Results}

The characteristic data of the patients are shown in Table I. The mean age of the patients was 38.71 \pm 7.68 years and the mean body mass index (BMI) was

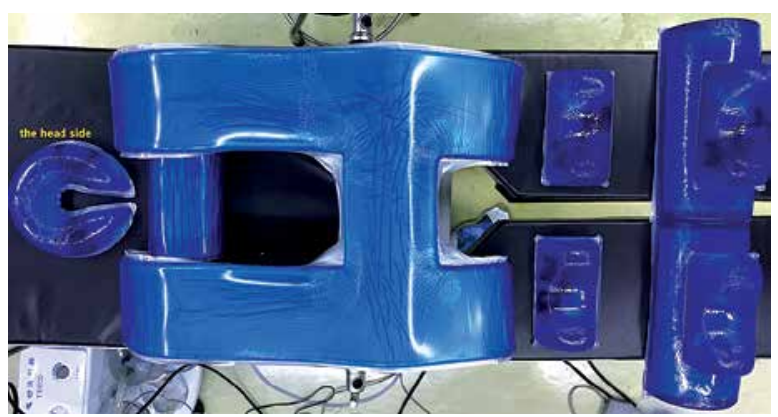

Photo 2. Gel pad under the patient

$22 \pm 2.02 \mathrm{~kg} / \mathrm{m}^{2}$. Five of the 17 patients had a cesarean section once, and 1 of the 17 patients had a history of two operations (cesarean section and laparoscopic cholecystectomy). The remaining patients had no history of surgery. The mean myoma volume in the ultrasound report was $121.99 \pm 125.24 \mathrm{~cm}^{3}$.

The intra- and postoperative surgical outcomes are shown in Table II. The mean operation time was $107.48 \pm 34.16 \mathrm{~min}$. The mean $\mathrm{Hb}$ decrease $48 \mathrm{~h}$ after the operation was $1.37 \pm 0.66 \mathrm{~g} / \mathrm{dl}$. The mean weight of the myoma was $183.88 \pm 144.29 \mathrm{~g}$. The mean VAS score $12 \mathrm{~h}$ and $24 \mathrm{~h}$ after surgery was $2 \pm 0.87$ and $1.18 \pm 0.73$, respectively. The mean postoperative hospital stay was $3.18 \pm 0.39$ days.

One patient was converted to TU-LESS. No other complications such as massive hemorrhage, infection or injury occurred.

The final histology was uterine leiomyoma in all of the cases.

\section{Discussion}

V-NOTES is a new method of gynecologic surgery. Since V-NOTES was carried out in our hospital in 

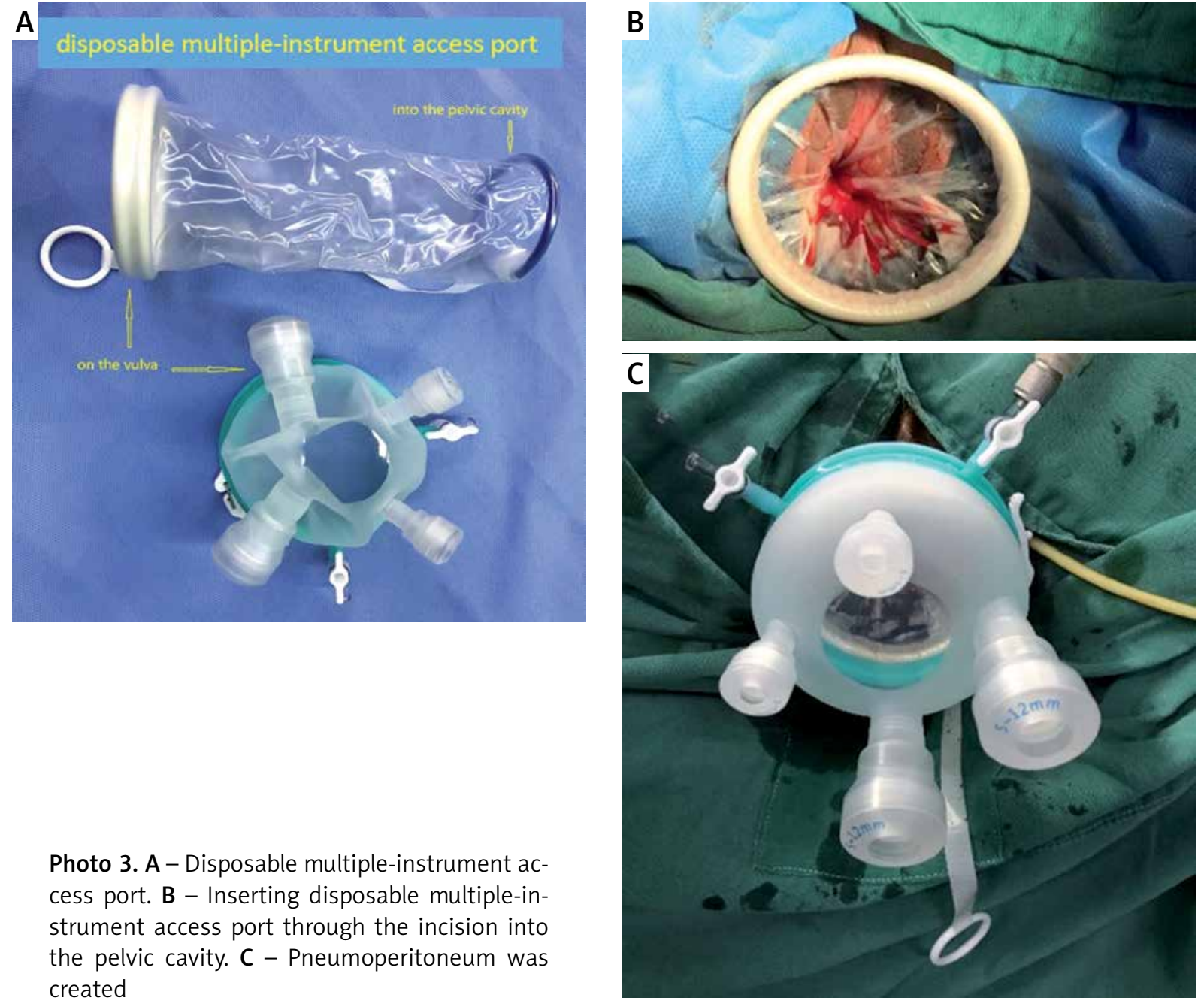

Photo 3. A - Disposable multiple-instrument access port. B - Inserting disposable multiple-instrument access port through the incision into the pelvic cavity. $\mathbf{C}$ - Pneumoperitoneum was created
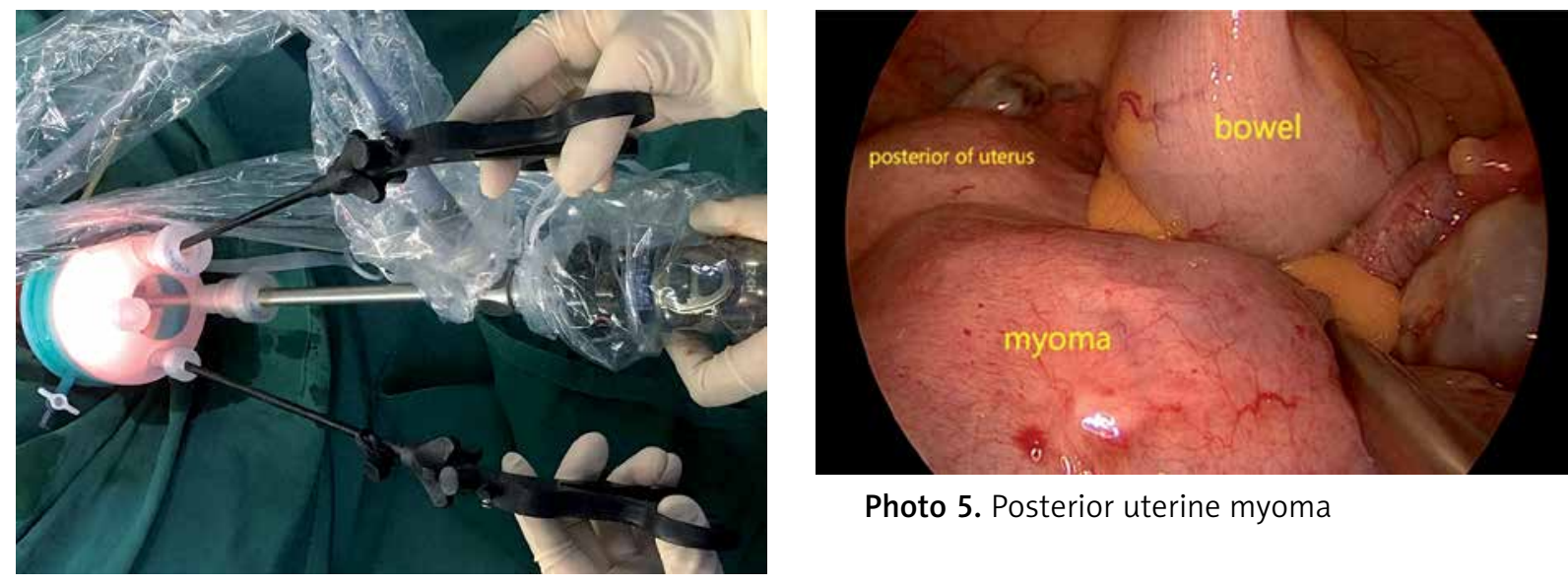

Photo 5. Posterior uterine myoma

Photo 4. Inserting endoscope and instruments through the disposable multiple-instrument access port 


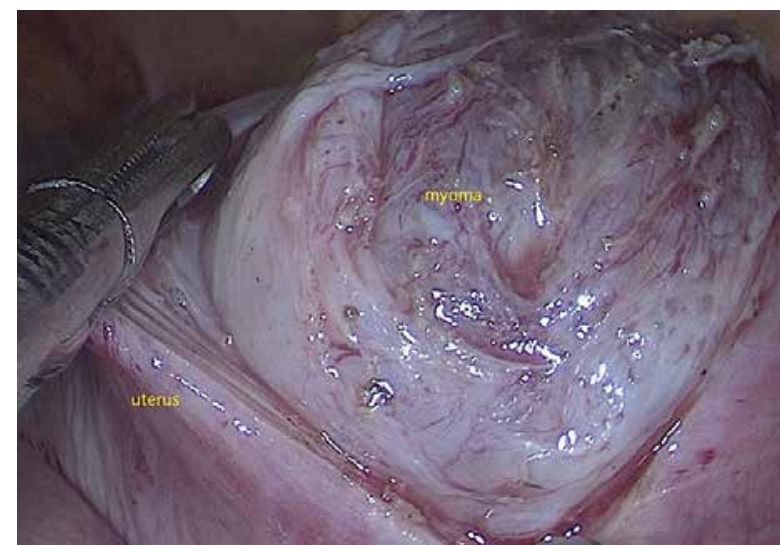

Photo 6. Enucleation of the myoma

2018, more than 200 cases of benign gynecological diseases had been treated by V-NOTES. Compared with conventional laparoscopy, V-NOTES eliminates the need of penetrating abdominal muscles and fas-

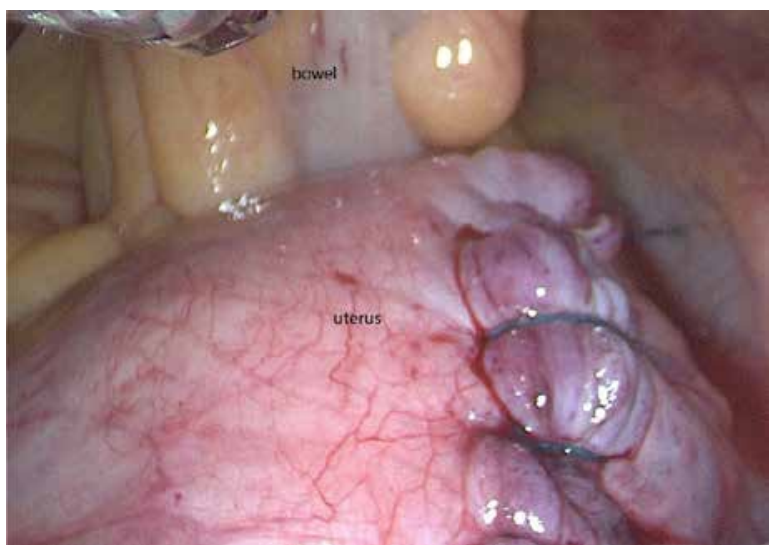

Photo 7. Repair of the uterine defect

cia and possible complications related to the trocar, and could achieve the best cosmetic outcomes [2]. Moreover, as the vaginal fornix is innervated by visceral nerves, pain felt by patients after the operation

Table I. Characteristics of patients

\begin{tabular}{|c|c|c|c|c|c|}
\hline Case no. & $\begin{array}{c}\text { Age } \\
\text { [years] }\end{array}$ & $\begin{array}{l}\text { Parity } \\
\text { (times) }\end{array}$ & $\begin{array}{l}\text { Previous abdominal } \\
\text { procedures } \\
\text { (times) }\end{array}$ & $\mathrm{BMI}\left[\mathrm{kg} / \mathrm{m}^{2}\right]$ & $\begin{array}{c}\text { Myoma volume in } \\
\text { ultrasound report } \\
{\left[\mathrm{cm}^{3}\right]}\end{array}$ \\
\hline 1 & 34 & 0 & 0 & 28.06 & 83.88 \\
\hline 2 & 42 & 1 & 1 & 22.15 & 71.99 \\
\hline 3 & 32 & 1 & 1 & 21.44 & 42.10 \\
\hline 4 & 49 & 1 & 0 & 23.44 & 139.97 \\
\hline 5 & 43 & 1 & 0 & 22.19 & 94.42 \\
\hline 6 & 49 & 1 & 0 & 22.35 & 514.00 \\
\hline 7 & 37 & 1 & 1 & 22.03 & 63.26 \\
\hline 8 & 47 & 1 & 0 & 20.70 & 42.48 \\
\hline 9 & 27 & 0 & 0 & 19.35 & 48.52 \\
\hline 10 & 37 & 1 & 0 & 22.96 & 331.27 \\
\hline 11 & 51 & 1 & 1 & 23.37 & 70.73 \\
\hline 12 & 32 & 2 & 1 & 22.31 & 70.97 \\
\hline 13 & 31 & 1 & 2 & 21.1 & 77.33 \\
\hline 14 & 41 & 1 & 0 & 22.77 & 68.94 \\
\hline 15 & 43 & 1 & 0 & 19.53 & 41.55 \\
\hline 16 & 27 & 0 & 0 & 19.78 & 219.71 \\
\hline 17 & 36 & 1 & 0 & 20.55 & 92.73 \\
\hline mean & 38.71 & 0.88 & 0.41 & 22.00 & 121.99 \\
\hline Standard deviation & 7.68 & 0.49 & 0.62 & 2.02 & 125.24 \\
\hline
\end{tabular}

$B M I-$ body mass index. 


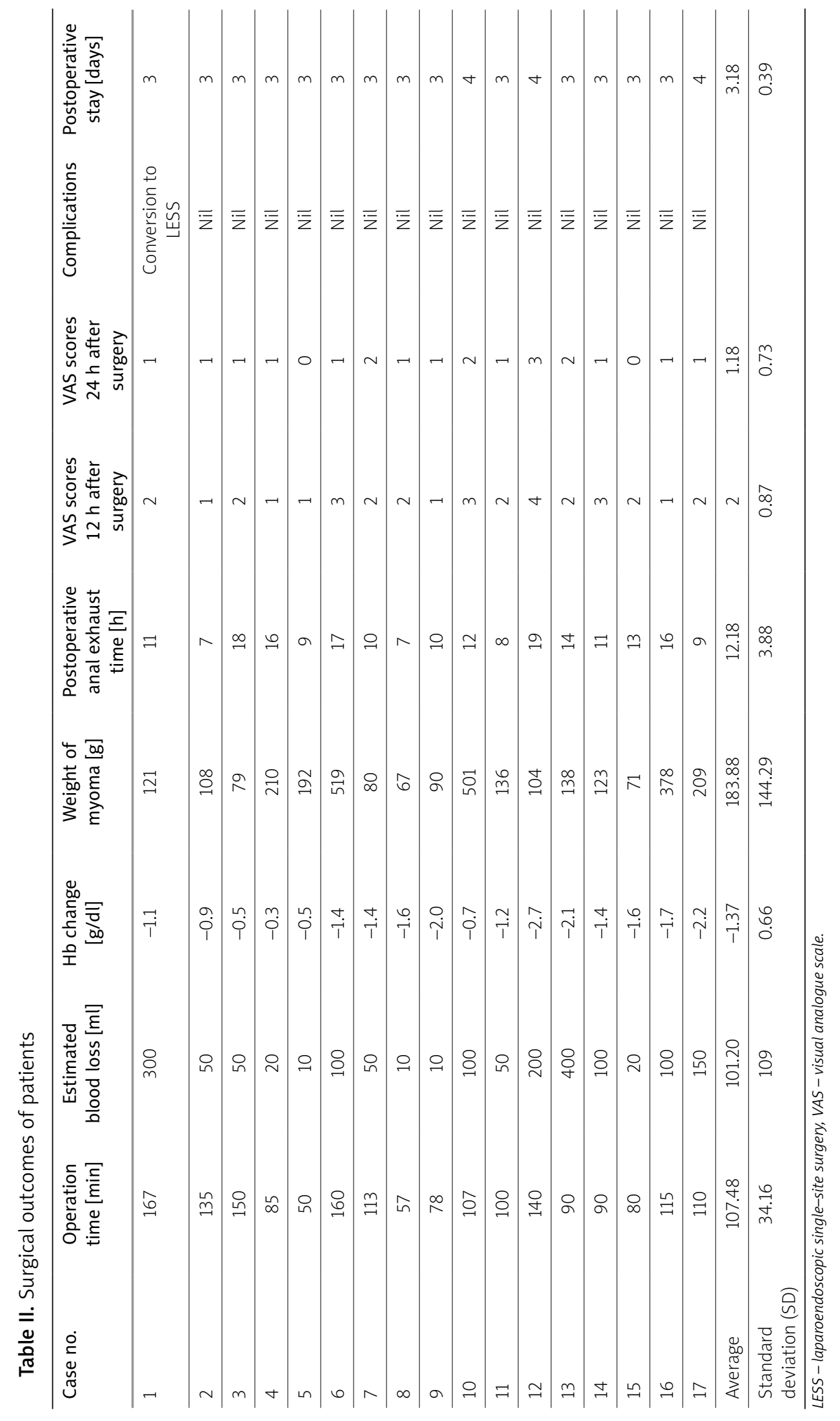


may be less than that of a traditional skin incision. The V-NOTES procedure overcomes the surgical and technical difficulties of conventional vaginal surgery by incorporating the advantages of endoscopic surgery [2]. Also it provides gynecologists an opportunity to observe the whole pelvis and abdominal cavity with direct vision, which greatly expands the scope of transvaginal surgery. In addition, the disposable incision retractor protecting the colpotomy makes retrieval of the specimen convenient and diminishes tumor spillage into the peritoneal cavity.

However, V-NOTES also has some limitations in gynecologic surgery, such as narrow space and inability of fully exposing the uterus during myomectomy. Meanwhile, the size and position of fibroids are diverse. Thus, the procedure via the vaginal route is technically challenging as a result of the restricted operative field. Thus, gynecologists have being reluctant to perform transvaginal myomectomy [3]. Therefore, there are few reports on V-NOTES myomectomy (Table III). Lee et al. in 2014 [4] explored 3 cases of V-NOTES myomectomy. They found that the procedure of V-NOTES myomectomy was more complex and potentially involved a greater risk than that of conventional laparoscopic myomectomy because of the relatively restricted vision. Therefore, they concluded that conversion to conventional laparoscopy was essential in a discrepant condition between preoperative image studies and operative findings. However, the follow-up reports confirm that V-NOTES is feasible in myomectomy. Liu et al. [5] in 2018 reported a case of V-NOTES anterior myomectomy (using video). In their opinion, V-NOTES broadens the application of vaginal surgery, and presents more advantages, especially for patients who have a large uterus with myoma or adenomyoma, because the vaginal flexibility enhances the extension of the colpotomy incision, allowing the laparoscopic instruments to reach a deeper area. Baekelandt [6] in 2017, reported 8 cases of V-NOTES myomectomy (using video), and opined that V-NOTES provided a new less invasive approach for the resection of myoma types 3-7.

As gynecological endoscopists know, in uterine myomectomy, whether by conventional laparoscopy, TU-LESS or V-NOTES, posterior uterine myoma is difficult to remove and suture because of the problem of exposure. In conventional laparoscopy and TU-LESS, we can solve this problem by using a uterine manipulator. But for V-NOTES uterine myomec-

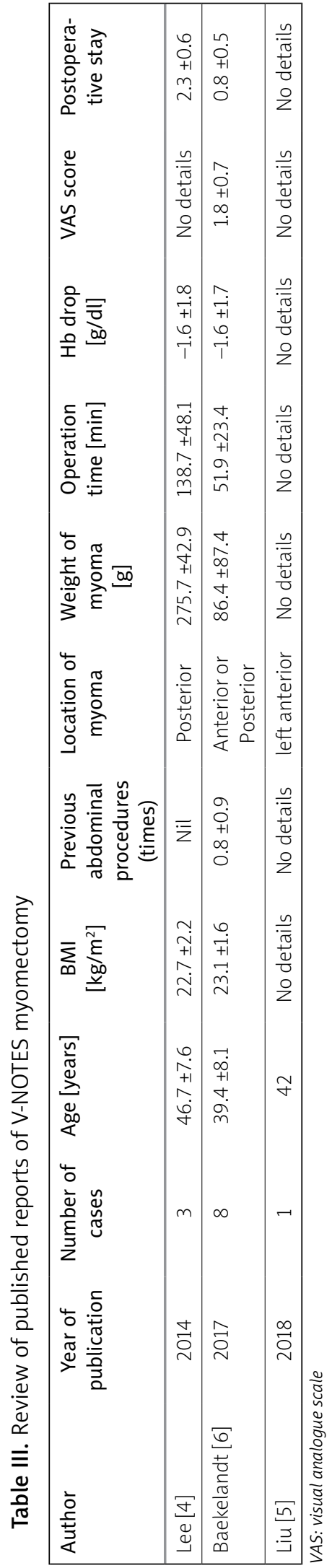


tomy, how can we expose the posterior wall of the uterus more effectively? In our study, patients were placed in the prone and Trendelenburg positions. Under the action of gravity, the organs including the uterus and intestine inclined upward and forward; thus a larger space was formed behind the uterus, facilitating the removal and suture of the uterine myoma. In our study, the largest case of uterine myoma was about $10 \mathrm{~cm}$ in diameter, so in our opinion the size of the myoma is not a contraindication in prone V-NOTES myomectomy. The average weight of the myoma in this study was greater than that reported by Baekelandt [6] and smaller than that reported by Lee et al. [4]. The average operation time was longer than that reported by Baekelandt [6] and shorter than that reported by Lee et al. [4]. HB decline after surgery was lower than that reported by Baekelandt [6] and Lee et al. [4]. The average VAS score at $12 \mathrm{~h}$ and $24 \mathrm{~h}$ after surgery belonged to the category of mild pain. From the above results, we can see that V-NOTES myomectomy in the prone position is feasible. Regarding the length of postoperative stay, our study is longer than that reported by Lee et al. [4] and Baekelandt [6], which may be related to different discharge standards, different patient perceptions and medical insurance systems.

Nevertheless, V-NOTES still has limitations in uterine myomectomy. For anterior myomectomy, we adopt a vaginal anterior fornix incision, which can only be performed on the anterior wall of the uterus, but one cannot observe the condition of the posterior wall of the uterus and it is also very difficult to operate on the lateral wall and the fundus of the uterus myomectomy. For posterior uterine myoma, we adopt a vaginal posterior fornix incision, but one cannot observe the anterior wall of the uterus, and it is also difficult to operate on the lateral wall and the fundus of the uterus myomectomy. Therefore, this technique is only suitable for excision of single anterior or posterior uterine fibroids. Among the 17 patients in this study, one patient transferred to TU-LESS just because of this limitation. After removal of the fibroid from the uterus, we did not remove the myoma from the pelvic cavity immediately. In the prone position, the myoma fell in front of the uterus under gravity and could not be detected. Finally, we changed the position of the patient to the lithotomy position and the fibroid was found in front of the uterus in the TU-LESS and removed through the vagina. So we believe that during the myomectomy by V-NOTES in the prone position, it is very important to take out the mass immediately after resection of the myoma. Perhaps, with the development of V-NOTES, an endoscope and instruments that can bend or rotate $360^{\circ}$ will be invented, and this limitation will eventually be resolved.

From our observations, V-NOTES in the prone position is safe and feasible. This position provides a wider field of view and a larger operating space for $V$-NOTES posterior uterine wall myomectomy. However, V-NOTES is an emerging surgical method and previous studies were mostly single-center retrospective studies, and there is a lack of prospective randomized controlled studies with large multi-center samples. For V-NOTES surgery in the prone position, we will further expand the sample size and conduct a prospective study to further confirm its safety and effectiveness.

\section{Conclusions}

Based on the previous reports V-NOTES myomectomy is a feasible approach to myomectomy, but it is difficult to preform due to the relatively narrow operating space, especially for posterior uterine fibroids. We adopt the prone position to transform the posterior myoma into a similar position to the anterior myoma, which can expand the operative space and reduce the difficulty of surgery. From our study, we can see that the prone position in V-NOTES myomectomy is safe and feasible. Perhaps it can be used as a standard position for posterior uterine myomectomy by V-NOTES.

\section{Acknowledgments}

Lu Huang and Li He contributed equally to the article.

\section{Conflict of interest}

The authors declare no conflict of interest.

\section{References}

1. Yoshiki N. Review of transvaginal natural orifice transluminal endoscopic surgery in gynecology. Gynecol Minim Invasive Ther 2017; 6: 1-5.

2. Kale A, Sarlibrahim B, Basol G. Hysterectomy and salphingoopherectomy by transvaginal natural orifice transluminal endoscopic surgery (NOTES): Turkish surgeons' initial experience. Int J Surg 2017; 47: 62-8. 
3. Li CB, Hua KQ. Transvaginal natural orifice transluminal endoscopic surgery (vNOTES) in gynecologic surgeries: a systematic review. Asian J Surg 2020; 43: 44-51.

4. Lee CL, Huang CY, Wu KY, el al. Natural orifice transvaginal endoscopic surgery myomectomy: an innovative approach to myomectomy. Gynecol Minim Invasive Ther 2014; 3: 127-30.

5. Liu J, Lin Q, Blazek K, et al. Transvaginal natural orifice transluminal endoscopic surgery myomectomy: a novel route for uterine myoma removal. I Minim Invasive Gynecol 2018; 25: 959-60.

6. Baekelandt J. Transvaginal natural-orifice transluminal endoscopic surgery: a new approach to myomectomy. Fertil Steril 2018; 109: 179.

Received: 26.02.2020, accepted: 20.04.2020. 